\title{
Human Face Recognition
}

\author{
Himani Sabharwal \\ Student at Department of \\ Electronics and \\ Communication \\ IGDTUW \\ New Delhi, India
}

\author{
Akash Tayal \\ Electronics and Communication \\ IGDTUW \\ New Delhi, India
}

\begin{abstract}
SIFT (scale invariant feature transform) being a feature extraction algorithm was initially used for object recognition, pattern recognition. But from past few years this algorithm is frequently being used for human face recognition based on feature extraction and matching. The twist here is that between feature extraction and its recognition I have used fuzzy clustering to create clusters so that we can localize the areas for feature point detection. As this recognition is based on a GUI system whose recognition rate is almost $99 \%$ $100 \%$ for face recognition and feature detection.
\end{abstract}

\section{Keywords}

SIFT, Fuzzy clustering, Image recognition, feature extraction.

\section{INTRODUCTION}

Image matching has always been an area of interest in fields related to computer vision, object recognition, pattern recognition, 3-D view images, scene recognition and many more such related areas. Now this image matching has created a new area of experiment and that is face recognition. As face recognition is mostly applicable in verifying authenticity, identification, bioinformatics. Thus mostly used for safety purpose and finding prime aspects of crime by gathering their information and past records. Thus face recognition, matching and feature extraction has become the hottest subject of image matching and analysis.

The basic requirement of image matching is to extract features of image and then which are used for finding a image in a dataset with which it matches mostly. Thus for finding features of human face I have used SIFT algorithm with a twist of applying clustering on an original image.

\section{RELATED WORK}

Recognition being the most interested field of researchers. SIFT has been mostly used for object recognition, pattern recognition etc. but now-a-days it's been used for face recognition using feature extraction. Thus face recognition is mostly used as an application of authenticity and bioinformatics. Earlier it's been used by various researchers out of which David lowe was first to present an improved version of this algorithm which was called as SIFT for video image matching in 2004 [8]. And the work related to it starts very back to reach the present day SIFT algorithm.

\section{THEORY}

Feature extraction for image recognition and matching is the key step in any pattern, object or image recognition. Extracting the features is based on minimization of the keypoints to attain the stable and reliable points on face. This approach is named as scale invariant feature transform (SIFT) because it transforms image data into scale-invariant coordinates to local features of input image[1]. There are four steps in which these features are extracted for this algorithm which are as follows [3] :

A.) Scale space extrema detection

B.) Keypoint localization

C.) Orientation assignment

D.) Keypoint descriptor

a.) Scale Space Extrema Detection - This is the first step of procedure. It is implemented to efficiently find interest points of facial features. These interest points are called keypoints. The keypoint detection is basically to identify positions and scales of interest points which can be viewed from differing views. The first essential function

$$
\mathrm{L}(\mathrm{x}, \mathrm{y}, \sigma)=\mathrm{G}(\mathrm{x}, \mathrm{y}, \sigma) * \mathrm{I}(\mathrm{x}, \mathrm{y})
$$

From [6,7,8] Convolution of Image $\mathrm{I}(\mathrm{x}, \mathrm{y})$ with gausian function $\mathrm{G}(\mathrm{x}, \mathrm{y}, \sigma)$ is a Gaussian blured image where $\mathrm{x}$ and $\mathrm{y}$ are coordinates of the input image $\mathrm{x}$ gives the distance from the horizontal axis ; y gives the distance from the vertical axis ,$\sigma$ acts as a scaling factor, $\mathrm{L}(\mathrm{x}, \mathrm{y}, \sigma)$ is the Gaussian blurred image or a spatial scale image. After getting the zoomed image by a factor of $\sigma$ and series of scale image could be obtained and then the difference of successive gausian blurred images are taken to find the keypoints. This difference is called as difference of Gaussian (DOG) images. The DOG gives the keypoints which are basically are maxima and minima of DOG that occurs at multiple scales i.e. neighborhood pixel values are compared to get keypoints. DOG of image,

$$
\mathrm{D}(\mathrm{x}, \mathrm{y}, \sigma)=\mathrm{L}(\mathrm{x}, \mathrm{y}, \mathrm{k} \sigma)-\mathrm{L}(\mathrm{x}, \mathrm{y}, \sigma)
$$

Thus DOG is the feature enhancement algorithm that involves the subtraction of 1 more blurred version of an original image $\mathrm{L}(\mathrm{x}, \mathrm{y}, \mathrm{k} \sigma)$ from another less blurred version of the same original image $\mathrm{L}(\mathrm{x}, \mathrm{y}, \sigma)$.

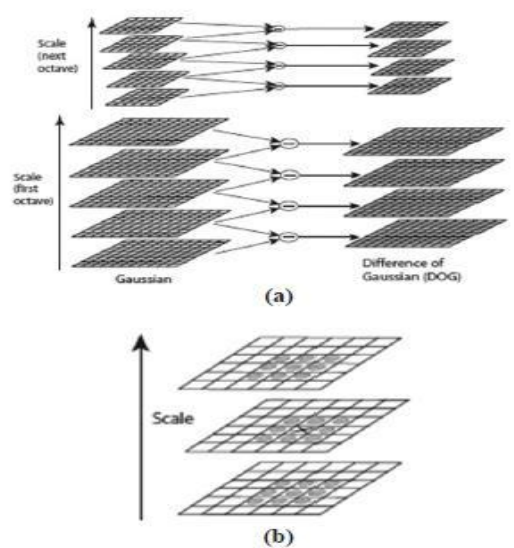

Figure 1. Representation of DOG calculation 
b.) Keypoint Localization - In this step as name says keypoint localization filters the keypoints of the processed image. From the above step when we detects the keypoints or interest points of image there are 2 types of keypoints - stable and other is unstable. Thus this step filter these unstable and unreliable keypoints which are sensitive to noise or disturbances. From the reference [8] aacording to taylor quadratic expansion, $\operatorname{DOG}(\mathrm{x}, \mathrm{y}, \sigma)$ low contrast interest points are removed.

c.) Orientation Assignment - This step finds the 2 types of orientation of every keypoint which finally got verified from above step. The 2 types of orientation are detecting magnitude and phase angle of the keypoint located. These are calculated via following formulae

$$
\begin{aligned}
& \mathrm{m}(\mathrm{x}, \mathrm{y})= \\
& \sqrt{\left[(\mathrm{L}(\mathrm{x}+1, \mathrm{y})-\mathrm{L}(\mathrm{x}-1, \mathrm{y}))^{2}+(\mathrm{L}(\mathrm{x}, \mathrm{y}+1)-\mathrm{L}(\mathrm{x}, \mathrm{y}-1))^{2}\right]} \\
& \theta(\mathrm{x}, \mathrm{y})=\tan ^{-1} \frac{[\mathrm{L}(\mathrm{x}, \mathrm{y}+1)-\mathrm{L}(\mathrm{x}, \mathrm{y}-1)]}{[\mathrm{L}(\mathrm{x}+1, \mathrm{y})-\mathrm{L}(\mathrm{x}-1, \mathrm{y})]}
\end{aligned}
$$

d.) Keypoint Descriptor - After implementing all above 3 steps - keypoints, locations and direction with amplitude are calculated. But these does not give assurance for various geometric invariance, illumination and view perspective. This step is to ensure that above problems does not create any other problem related to stable and reliable keypoint detection of image. Steps involved are :

- $\quad 16 \times 16$ pixel field is selected and is divided into 16 subfields of $4 \times 4$;

- Now according to step (C.) $\mathrm{m}(\mathrm{x}, \mathrm{y})$ and $\theta(\mathrm{x}, \mathrm{y})$ is calculated for every subfield. $\Theta(x, y)$ gives the direction distribution in the range from $(0,2 \pi)$ through which statistical histogram is drawn.

- According to the statical histogram of subfields, 8 direction descriptors are calculated.

- Feature description for all the subfield is calculated by connecting the direction descriptions of all subfields, so the length of feature description is 16 (total of the direction descriptions) $x 8=128$. And finally feature descriptor is normalized.

\section{HOW FACE RECOGNITION SYSTEM WORK}

This system works in a particular way. It's module can be viewed as follows :

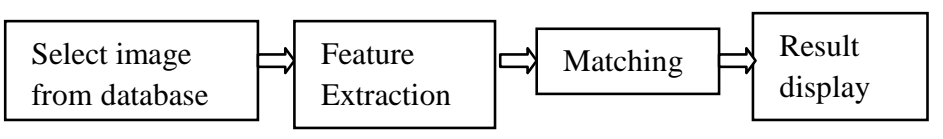

Figure 2 Face recognition module

\section{1) Module description :}

a.) Database :- The database of images choosed for this experiment is saved in pgm format. The pgm format is basically a portable graymap format which is a compact form of storing images in grayscale file format. This consists of 160 images in total to perform this experiment.

b.) Feature Extraction :- The feature extraction of this system is based on SIFT algorithm. This algorithm does not detect the section or area of features but only detects them in the form of feature points or may be said as interest points.

c.) Matching :- This module tries to find the image which is being loaded as input image for feature extraction. And when the image is found it is displayed on the axes.

\section{2) GUI System :}

In this paper, to display the implementation of my work a GUI is prepared. This GUI consists of :

a.) popup buttons - This system consists of 3 popup buttons. These are - load image, create database, matching. When the load image popup button is pressed the browser opens a drive from where we can select an image. Pressing a create database will create a database of feature extracted for every image of database with their display on separate gui window along with the same GUI system. Last one is matching popup button that will display the clean image with which loaded image got matched.

b.) Axes - In this gui system 2 axes are used. One to display input image or loaded image and second, for the image with which input image got matched.

c.) Edit text box - This edit text box is used to display the number of image loaded and being matched with.

The GUI system which is prepared for this experiment is as follows:

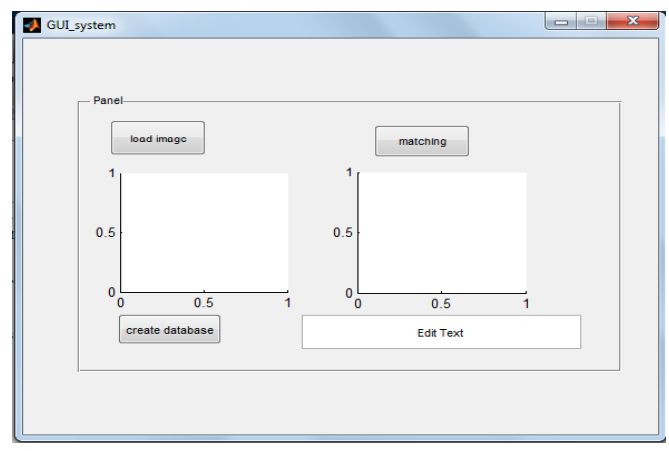

Figure3: GUI system prepared

4.2) Proposed method :

In my human face recognition gui system the loaded image is original one but when features are extracted or we may say when database of features to be extracted is created then a clustered image of original image was produced and finally when image matching is to be done then again the clear and original image was loaded if the input loaded image gets matched to the database of 160 images. Along with all the above description this gui system of human face recognition also displays the folder number with which it gets matched. 


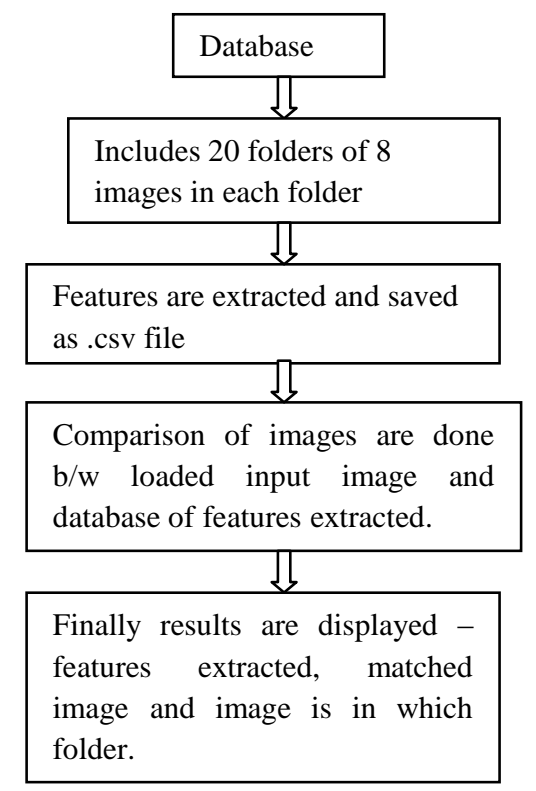

\section{RESULT AND CONCLUSION}

In this experiment a database of images are used which are saved in pgm format. These images are categorized in 20 folders each containing 8 images. Thus total database consist of 160 images. Features of all 160 images were deduced and then they were matched in the same folder.

The result contains one more part that displays the number of folder from which it was selected or is present in the folder. As already explained above this recognition system is based on GUI. The result of this system is as follows:

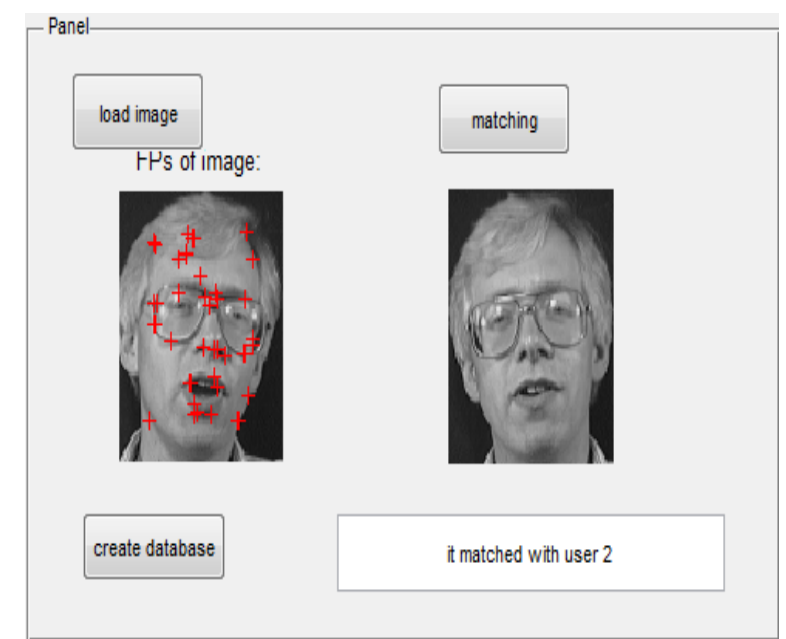

Figure 4: GUI result representation of face recognition System.
This system is doing 3 things simultaneously - feature extraction, matching, creating database of images and identifying the number of folder in which image was stored. As showed in above figure.

\section{ACKNOWLEDGMENTS}

This work was supported by Mr. Akash Tayal under his guidance at IGDTUW. I would like to thank him for suggestions in improvement to both the content and work of this paper.

\section{REFERENCES}

[1] David G. Lowe, "Distinctive image features from scaleinvariant keypoints," in international journal of computer vision, January 5, 2004.

[2] Wenyu Chen, Yanli Zhao , Wenzhi Xie and Nan Sang, "An Improved SIFT Algorithm for Image FeatureMatching,",

[3] David G. Lowe, "Object Recognition from Local ScaleInvariant Features," Proc. of the International Conference on Computer Vision, Corfu (Sept. 1999).

[4] Dilbag Singh, " Human emotion recognition system," in august 2012 in MECS, DOI 10.5815/ijigsp.2012.08.07.

[5] Shail Kumari Shah, " A survey of facial expression recognition methods," in IOSR journal of engineering (iosrjen), ISSN (e): 2250-3021, ISSN (p): 2278-8719 Vol. 04, Issue 04 (April. 2014), ||V3\| PP 01-05.

[6] Qing Zhang and Minho Lee,"Fuzzy-GIST for emotion recognition in natural scene images," in 2009 IEEE $8^{\text {th }}$ international conference on development and learning.

[7] Han Yanbin, Yin Jianqin and Li Jimping, "Human Face Feature Extraction and Recognition Base on SIFT," in 2008 international symposium on computer science and computational technology, IEEE.

[8] David G. Lowe, "Local feature view clustering for 3d object recognition," Proc. of the IEEE Conference on Computer Vision and Pattern Recognition, Kauai, Hawaii (December 2001). 